\title{
Management of depression in later life
}

\author{
Robert Baldwin \& Rebecca Wild
}

Abstract Both antidepressants and psychological treatments are effective in the management of late-life depression. Nevertheless, there remains a considerable challenge to improve the prognosis for depression in older people. Endlessly increasing the range of antidepressants does not seem to be the answer, so attention is turning to new combinations of treatments and new ways of delivering care and improving treatment uptake. Collaboration between specialist and primary care, case management and multifaceted interventions are currently the most exciting prospects. There is good evidence for the role of both medication and psychological treatment in keeping the patient well after recovery.

Depressive disorder is the most frequent psychiatric illness of older people (Beekman et al, 1999). It has a negative impact on quality of life, adds significantly to disability from physical disorder (Penninx et al, 2000) and is the leading cause of suicide in older people. It is also an independent predictor of mortality (Cuijpers \& Smit, 2002). Although effective treatment exists, depression is not well recognised and often not optimally managed (Baldwin et al, 2002). This article updates an earlier review of the subject (Katona, 1996).

\section{Box 1 Factors to take into account when} diagnosing late-life depression

Altered symptoms in late-life depression

- Reduced complaint of sadness

- Hypochondriasis and somatic concern instead of sadness

- Poor subjective memory or a dementia-like picture

- Late-onset neurotic symptoms (marked anxiety, obsessive-compulsive or hysterical symptoms)

- Apathy and poor motivation

Symptoms that may be hard to interpret because of comorbid physical disorder ${ }^{1}$

- Anorexia

- Weight loss

- Reduced energy

1. For a discussion, see Koenig et al (1997).

\section{Prevalence in older people}

In a meta-analysis the prevalence of clinically significant depression among older people living in the community was $13.5 \%$ (Beekman et al, 1999). Important determinants of prevalence are disability and handicap so, unsurprisingly, rates are at least doubled among patients in hospital or nursing homes. There are possible neurobiological links between depression and stroke, Parkinson's disease and the dementias (vascular dementia having the highest rate of comorbid depression), but also a high rate of depressive symptoms in a range of chronic medical disorders, including heart disease (Ariyo et al, 2000) and chronic obstructive pulmonary disease (Yohannes et al, 1998).

\section{Assessing depression in later life}

Special factors to take into account when assessing depression in older people are listed in Box 1. The skills of history-taking, conducting a mental state examination, physical evaluation and organisation of relevant investigations are no less important than in other branches of psychiatry. Given the increased likelihood of finding an organic condition contributing to depression with advancing age, these skills are arguably more important. Boxes 2 and 3 list the more important causes of organic depressive episodes.

Robert Baldwin has been a consultant in old age psychiatry at Manchester Royal Infirmary since 1985 (Manchester Mental Health \& Social Care Trust, York House, Manchester Royal Infirmary, Oxford Road, Manchester M13 9BX, UK. Tel: +44 (0)161 276 5303; fax: +44 (0)161 276 5317; e-mail: Robert.Baldwin@man.ac.uk) and Honorary Professor of Psychiatry at the University of Manchester since 2000. His main research interest is mood disorders in later life, on which he has published widely. Rebecca Wild trained as a specialist registrar in Greater Manchester and is now a consultant in old age psychiatry at Bolton Royal Hospital in Lancashire. 
Box 2 Medications that may cause organic depression

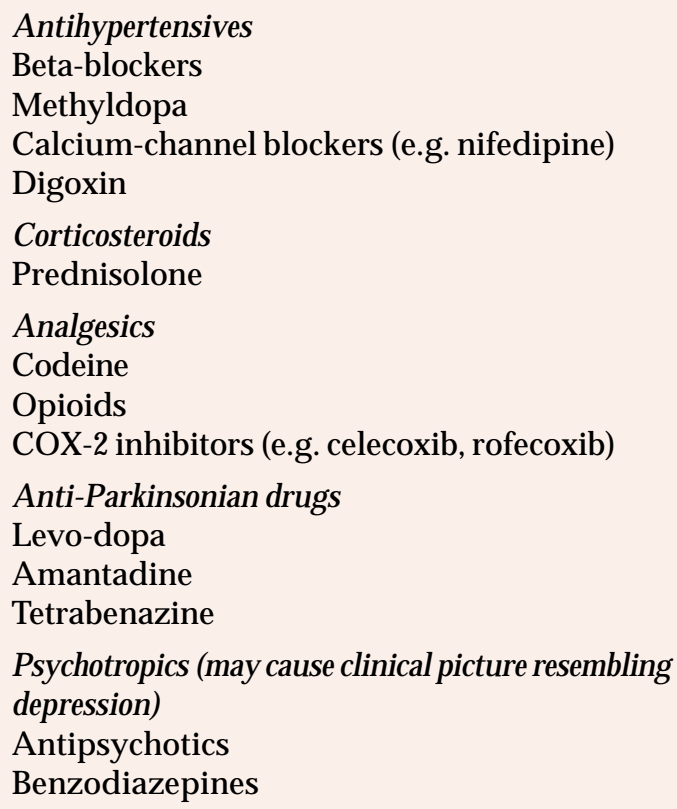

\section{Screening}

The most widely validated screening instrument is the Geriatric Depression Scale, introduced in 1983 (Yesavage et al, 1983). Short and long versions and translations of the scale are available at http:// www.stanford.edu/ yesavage/GDS.html. The 15-item version (with its 4 - and 5-item derivatives) is shown in Box 4. The 5-item version has recently been validated in a sample comprising individuals living in the community, hospitalised patients and nursing-home residents (Rinaldi et al, 2003).

\section{Types of depression in older people}

\section{Minor and major depression}

Although $13.5 \%$ of older people are significantly depressed, the prevalence of depressive episode (major depression) is much lower, at around 2\% (Beekman et al, 1999). Minor depression is one term used to describe the less severe depression affecting the remaining group. Recent evidence suggests that minor and major depression in older people share similar risk factors and that in population terms the adverse consequences of minor depression lie midway between major depression and not having depression at all (Cuijpers \& Smit, 2002). Minor depression is perhaps characterised by more cognitive symptoms of depression and fewer somatic ones. There is a striking lack of data about effective treatment. In one study, paroxetine was moderately effective for both persistent minor depression and dysthymia in patients with functional impairment, but problem-solving treatment was not (Williams et al, 2000). However, there was marked site variation in the quality of the problemsolving treatment. In another study (Mossey et al, 1996) a treatment similar to the problem-solving treatment was effective in patients with subthreshold depression with medical comorbidity.

\section{Vascular depression}

The clinical features of this proposed new subtype include apathy, psychomotor retardation, poor executive function on cognitive testing, less depressive thinking (such as guilt or unworthiness) and a late age at onset. The basis is thought to be ischaemically induced white matter changes (Baldwin \& O'Brien, 2002). This subtype is probably less responsive to antidepressant drugs (Simpson et al, 1998), but patients may recover with electroconvulsive therapy (ECT), although with an increased risk of post-treatment delirium.

\section{Prognosis}

Cole \& Bellavance (1997a) reported that after an index episode about $60 \%$ of secondary care patients either

\section{Box 3 Physical disorders that may cause} organic depression

Endocrine/metabolic

Hypo- and hyperthyroidism

Cushing's syndrome

Hypercalcaemia (primary hyperparathyroidism or carcinoma)

Pernicious anaemia

Folic acid deficiency

Organic brain disease

Cerebrovascular disease/stroke

Tumours of the central nervous system

Parkinson's disease

Alzheimer's disease

Systemic lupus erythematosus

Occult carcinoma

Pancreas

Lung

Chronic infections

Neurosyphilis

Brucellosis

Herpes zoster 


\section{Box 4 The 15-item Geriatric Depression Scale, also showing questions for the 4- and 5-item scales}

Instructions: Choose the best answer for how you have felt over the past week.

1 Are you basically satisfied with your life?

Yes/No

(No)

2 Have you dropped many of your activities and interests?

Yes/No

(Yes)

Do you feel your life is empty?

4 Do you often get bored?

5 Are you in good spirits most of the time?

Yes/No

(Yes)

Yes/No

(Yes)

Yes/No (No)

Are you afraid something bad is going to happen to you?

Yes/No

(Yes)

Do you feel happy most of the time?

Yes/No

(No)

8 Do you often feel helpless?

Yes/No

(Yes)

9 Do you prefer to stay at home, rather than going out and doing new things?

10 Do you feel you have more problems with your memory than most?

Yes/No

(Yes)

Yes/No (Yes)

11 Do you think it is wonderful to be alive now?

Yes/No

(No)

12 Do you feel pretty worthless the way you are?

Yes/No

(Yes)

13 Do you feel full of energy?

14 Do you feel that your situation is hopeless?

Yes/No

Yes/No

(No)

15 Do you think most people are better off (in their lives) than you are?

Yes/No

Questions 1, 2, 6 and 7 make up the 4-item version.

Questions 1, 4, 8, 9 and 12 make up the 5-item version.

The answers shown in parentheses indicate possible depression.

Possible cut-offs: $\geq 5$ for the 15-item version; $\geq 2$ for the 4-item and 5-item versions.

remained well or had treatable relapses. About 1 in 5 developed chronic symptoms. Outcome for individuals living in the community was worse. In another meta-analysis of depressed patients in acute medical and surgical care, only about 1 in 5 recovered (Cole \& Bellavance, 1997b). Low rates of detection and undertreatment may partly explain these poor results (Baldwin, 2000).

Katona's statement in an earlier review (Katona, 1996) still holds true: depression in old age is associated with chronicity and a high risk of relapse after recovery. However, this is just as true for younger adults, prompting current interest in managing depressive disorder within a chronic disease model (Rost et al, 2002).

Mortality is high in older patients with depression, largely because of concurrent physical disorder (Tuma, 2000).

\section{Principles of management}

The management of depressive disorder in older people should be multimodal (involving physical and psychological modalities along with social interventions) and multidisciplinary (with help from specialists such as nurses, social workers and occupational therapists, and help as needed from dieticians, speech and language therapists and podiatrists). Some broad goals of treatment and ways of achieving them are shown in Table 1 (Baldwin et al, 2002). Handicap, the social disadvantage caused by illness, is closely associated with depression in old age. Thus, social factors such as the availability of transport and crime reduction may have very positive effects on reducing rates of depression.

Treatments that work are the same as for younger adults: antidepressants, psychosocial and psychological interventions, or combinations of these (Anderson et al, 2000), and ECT.

\section{Drug treatment}

Pharmacodynamics and pharmacokinetics alter with ageing and are also affected by concomitant medication and physical illness. Older people exhibit marked inter-individual variation in how medication is metabolised. This can lead to difficulty in predicting the target dose. The adage 'start low, go slow' then applies. This is less of a problem with the newer antidepressants, for which the starting dose and the therapeutic dose are identical. However, therapeutic trials often exclude the very old and the very frail, so that our knowledge is incomplete. Although many newer antidepressants require no dose adjustments for older patients, impaired renal or hepatic function necessitates caution with all antidepressants. Table 2 lists the main antidepressants, starting doses and average dosages for older patients by consensus (Alexopoulos et al, 2001; Baldwin et al, 2002), along with side-effect profiles. 


$\begin{array}{ll}\text { Table } 1 \text { Management principles } & \text { Ways to achieve goals } \\ \begin{array}{ll}\text { Goal } \\ \text { Reduction of risk of suicide or self-neglect }\end{array} & \begin{array}{l}\text { Carry out risk assessment and monitor risk (review regularly) } \\ \text { Ensure prompt referral of urgent cases (in primary care and on medical } \\ \text { wards) to a specialist }\end{array} \\ \begin{array}{ll}\text { Remission of all depressive symptoms } \\ \text { (residual symptoms increase the risk }\end{array} & \begin{array}{l}\text { Provide appropriate treatment (antidepressants, psychological } \\ \text { treatment or electroconvulsive treatment) }\end{array} \\ & \begin{array}{l}\text { Give the patient and supporters/carers timely education about } \\ \text { depression (e.g. Depression in Older Adults leaflet, free at http:// }\end{array} \\ & \text { www.rcpsych.ac.uk/info/help/doa/index.htm) } \\ \text { Optimise function } & \begin{array}{l}\text { Treat comorbid physical health problems } \\ \text { Attend to sensory deficits } \\ \text { Review medication }\end{array} \\ & \text { Enable practical support } \\ & \text { Facilitate access to appropriate helping agencies ('sign-posting') } \\ & \text { Encourage the patient to stay on medication when recovered } \\ \text { Continue treatment for 12 months after recovery (see text) }\end{array}$

Older patients take longer to recover. Whereas 4 weeks may be a sufficient time in which to see a response in younger adults, $6-8$ weeks or longer may be more typical for older patients. Nearly 20 antidepressant drugs are currently available for use in the UK. With such a wide variety of antidepressants it is best to tailor the drug to the patient, the choice being determined by symptom profile, tolerance and the likelihood of drug interactions or side-effects.

\section{Efficacy}

A recent Cochrane systematic review (Wilson et al, 2001) has demonstrated that antidepressants are an effective treatment for elderly patients with major depression and it revealed no important differences between classes of antidepressants. However, only 17 trials met inclusion criteria and there were few placebo-controlled studies of newer antidepressant drugs. Most recent trials have been head-to-head comparisons of older and newer drugs. These show a trend for selective serotonin reuptake inhibitors (SSRIs) and venlafaxine to be preferred because of a favourable adverse effects profile (Katona \& Livingston, 2002). Lastly, there is some evidence from trials involving patients of mixed ages (Anderson et al, 2000) that SSRIs may be less effective than tricyclics in in-patients with melancholic depression. Wilson et al (2001) concluded that there was insufficient evidence to recommend low-dosage antidepressant treatment in depressive episode in primary care. More research is needed.

\section{Comorbidity}

Patients with psychotic depression usually require a combined approach with the addition of antipsychotics or ECT. Physical comorbidity is common in older patients. A meta-analysis of antidepressant therapy $v$. placebo, although not exclusively in older patients, showed a number-needed-to-treat (NNT) of 4 for patients with depression and a range of physical disorders (Gill \& Hatcher, 1999). A similar figure is reported in the reviews of Wilson et al (2001) and Katona \& Livingston (2002) in older depressed patients without comorbidity. MacHale has outlined practical therapeutic strategies for patients with physical comorbidity in an earlier article in APT (MacHale, 2002).

\section{Tolerability and side-effects}

An important principle when treating older people is to remember that they have less physiological reserve and are more likely to lose homeostasis rapidly. For example, severe depression in an older person may quickly lead to dehydration, weight loss and even pressure sores. Likewise, older people are more susceptible to medication side-effects.

Anticholinergic side-effects of tricyclics, such as constipation, blurred vision and dry mouth, can be very troublesome for elderly patients, and postural hypotension, cardiac arrhythmia or overdose can be very dangerous. Delirium can occur, but is more likely in patients who are also acutely medically unwell. Lofepramine is a second generation tricyclic which is less likely to cause these adverse effects. 
Table 2 Average dosages and side-effect profiles of antidepressants (after Baldwin et al, 2002)

\begin{tabular}{|c|c|c|c|c|c|}
\hline & Anticholinergic & Antihistaminic & $\begin{array}{c}\alpha_{1} \text {-adrenergic } \\
\text { blocking }\end{array}$ & $\begin{array}{l}\text { Starting } \\
\text { dose }(m g)\end{array}$ & $\begin{array}{c}\text { Average } \\
\text { daily dose }(m g)\end{array}$ \\
\hline Amitriptyline & ++++ & ++++ & ++++ & $25-50$ & $75-100$ \\
\hline Imipramine & +++ & ++ & +++ & 25 & $75-100$ \\
\hline Nortriptyline & +++ & ++ & ++ & $10-30$ & 75-100 \\
\hline Dosulepin (dothiepin) & +++ & ++ & ++ & $50-75$ & $75-150$ \\
\hline Lofepramine & + & + & + & $70-140$ & $70-210$ \\
\hline Trazodone & 0 & +++ & + & 100 & 300 \\
\hline Fluvoxamine & $0 /+$ & $0 /+$ & 0 & $25-100$ & $100-200$ \\
\hline Sertraline & $0 /+$ & 0 & 0 & $25-50$ & 50-100 \\
\hline Fluoxetine & $0 /+$ & 0 & 0 & 10 & 20 \\
\hline Paroxetine & $0 /+$ & 0 & 0 & $10-20$ & $20-30$ \\
\hline Citalopram & $0 /+$ & 0 & 0 & $10-20$ & $20-30$ \\
\hline Escitalopram & 0 & 0 & 0 & $5-10$ & 20 \\
\hline Phenelzine & $0 /+$ & 0 & ++ & 15 & $\begin{array}{c}30-45 \\
\text { (divided doses) }\end{array}$ \\
\hline Moclobemide & $0 /+$ & 0 & 0 & 300 & $300-400$ \\
\hline Venlafaxine & $0 /+$ & 0 & $0 /+$ & $25-75$ & $75-150$ \\
\hline Mirtazapine & 0 & ++ & 0 & 7.5-15 & $15-30$ \\
\hline
\end{tabular}

It is more expensive than the older tricyclics, but no more expensive than SSRIs. The latter drugs are not cardiotoxic and are not usually lethal in overdose, but they have other undesirable side-effects. Gastrointestinal symptoms are well recognised, but there is growing concern about gastrointestinal haemorrhage, particularly in elderly patients (van Walraven et al, 2001). Caution should be exercised in patients treated with non-steroidal antiinflammatory drugs or aspirin. Sertraline and citalopram have the least potential for drug interactions. Epilepsy is a caution for the use of antidepressants.

Discontinuation symptoms may occur with all classes of antidepressants after 8 or more weeks of treatment. They are more common and severe with antidepressants that have a short half-life. Inappropriate antidiuretic hormone (IADH) secretion is often linked to SSRIs, but may occur as a side-effect of all classes of antidepressants. There is a paucity of systematic data, but increased age, female gender and drugs that lower sodium levels are all risk factors (Kirby \& Ames, 2001). Symptoms often (but not invariably) occur when the blood serum level falls below $130 \mathrm{mmol} / 1$. Symptoms of IADH, which include lethargy, fatigue and sleep disturbance as well as muscle cramps and headaches, overlap with those of depression. A high level of suspicion is needed.

Moclobemide is well tolerated by older people. Although a special diet is not required, patients should be aware of the drug interactions with painkillers and other antidepressants. Venlafaxine is an effective drug in this age group and is generally well tolerated, particularly if the dose is increased slowly. The main side-effects of nausea and gastrointestinal disturbance tend to be transitory. Venlafaxine does have an effect on blood pressure, but studies have found that elderly people (at least, those that are fit) are no more susceptible to this problem than younger patients. There is some evidence to support the use of venlafaxine in patients who have not responded to SSRIs, although not specifically in older patients (Poirier \& Boyer, 1999).

The SSRIs have minimal impact on cognitive function in older patients with depression and there is also evidence that SSRIs and lofepramine cause less impairment than the older tricyclics in cognitive skills relevant to driving.

\section{Psychological interventions}

Older patients with depression are rarely offered a psychological intervention. Changing attitudes and increased awareness are likely to alter this. In a large primary-care-based study in which a treatment choice was offered to older patients with depression, $50 \%$ expressed a preference for a psychological approach over drugs (Unützer et al, 2002). Additionally, there is emerging evidence that for older adults with mild-to-moderate depressive episodes a psychological intervention is as effective as medication (McCusker et al, 1998; Pinquart \& Sorensen, 2001). Whether services are adequately configured to meet the need is more questionable. Nevertheless, in England, the National Health Service Executive's strategic review of psychotherapy services has 
endorsed the need for accessibility and equity across patient groups (National Health Service Executive, 1996). General areas of importance when working psychodynamically with older people have been addressed in a previous APT article (Garner, 2002).

Cognitive-behavioural therapy (CBT) is the bestestablished treatment in depression and good evidence exists for its effectiveness in older adults (Thompson et al, 2001). However, most studies have looked at its use in cognitively intact and medically stable patients and so its effectiveness outside this patient group is not fully established. Small studies and case reports have indicated that CBT can be adapted for use with physically frail patients and those with mild cognitive impairment, but further research is needed. Interpersonal therapy is also effective in relapse prevention (Reynolds et al, $1999 a, b)$. There is a smaller but developing evidence base for problem-solving treatment (Arean et al, 1993; Unützer et al, 2002). In major depression, a combination of antidepressants with psychotherapy is more effective than either of these treatments alone, especially in relapse prevention (Reynolds et $a l, 1999 b$; Thompson et al, 2001). Psychoeducational techniques have also been used in this age group with good effect. For example, in one recent study a group course on coping with depression and anxiety was effective in reducing scores on the Geriatric Depression Scale (Schimmel-Spreeuw et al, 2000).

Lastly, family therapy has been successfully adapted for use with older adults, including those with depression (Benbow et al, 1990), but no controlled data have been published regarding efficacy.

\section{Electroconvulsive therapy}

Electroconvulsive therapy remains the most effective treatment available for severe depression, with a recovery rate in the region of $80 \%$. It is well tolerated, even by very elderly people (Tew et al, 1999). Its use is generally reserved for when there is a threat to life due to insufficient dietary intake or suicidal behaviour, or if treatment with antidepressants has been ineffective. There is evidence that it is particularly effective in psychotic depression (Baldwin et al, 2002). There are no absolute contraindications, but a review of medical risk by a senior anaesthetist is advisable. Wherever possible hypertension and cardiac failure should be treated optimally before ECT. It should be avoided in the first 3 months following a myocardial infarction or stroke. Elderly patients are more likely to suffer postECT confusion and cognitive impairment and therefore this should be carefully monitored during treatment. Memory impairment is often worse with bilateral electrode placement although the response to bilateral treatment may be more rapid.

\section{Other treatments}

Rapid transcranial magnetic stimulation (rTMS) is a promising new treatment for depression. The early evidence suggests that it may be less effective in older adults, especially in the presence of even mild frontal atrophy (Manes et al, 2001). St John's Wort, a herbal remedy, is popular but lacks a satisfactory evidence base. Epidemiological data support a link between depressive symptoms and lack of exercise in older people (Baldwin et al, 2002), but data have not been published on the use of exercise as a treatment for late-life depression.

In an important study designed to replicate models of enhanced care (also known as 'stepped care') developed for use with younger patients in primary care, Unützer et al (2002) showed that a multifaceted intervention for depression was more effective than usual care for older adults. The model chosen emphasised case management, the use of antidepressants and/or problem-solving treatment, and improved links between primary and specialist care.

\section{Treatment-resistant depression}

Although definitions of treatment resistance vary, at least $30 \%$ of elderly patients with depression do not respond to first-line treatment with an antidepressant (McCusker et al, 1998). The initial steps are to ensure that a medical cause for depression has not been overlooked, that the patient tolerates and takes the medication and that the dosage is optimal.

New data suggest that if the patient has shown little or no response (in objective terms less than a quarter improvement on an appropriate rating scale) within the first 4 weeks at therapeutic dosage, recovery is unlikely (Mottram et al, 2002). The best course is to change to an antidepressant of a different class. If, however, there has been a partial response, the clinician is faced with a choice. Other things being equal, because older patients take longer to recover (Anderson et al, 2000), waiting and supporting the patient may be a reasonable course of action. Otherwise, the advantages and disadvantages of augmentation and substitution, the two main strategies open to the clinician, are outlined in Box 5 (augmentation refers to adding another treatment to the original medication).

Clinicians in the UK tend to be 'swappers' - from one class of antidepressant to another. The use of 
Box 5 Factors favouring augmentation or substitution regimens (after Mulsant et al, 2001)

Augmentation

- Maintains improvement already achieved in partial responders

- Prevents delays associated with withdrawal of existing drug

- May have synergistic effects on two neurotransmitter systems

- Allows current partially successful agent to be continued for longer

Substitution

- Avoids potential drug-drug interactions

- Simpler regimen

- Lower costs

- Easier to attribute side-effects and understand tolerance

non-reversible monoamine oxidase inhibitors as an option has gone out of favour, although there was an early evidence base for their efficacy. Another approach, of combining a tricyclic with an SSRI, has given way to the newer dual-acting antidepressants such as venlafaxine or combinations such as SSRIs with mirtazapine. Both have some evidence to support their use in resistant depression (Anderson, 2003), although not specifically in older people. The high doses of venlafaxine sometimes recommended are not always tolerated by older patients. Likewise, augmentation with lithium has a reasonable evidence base, but tolerability can be a significant problem in older patients. Serum monitoring is required.

\section{Maintenance treatment}

How long to continue with maintenance therapy is not yet clear. A recent expert consensus statement suggests that after a first episode of major depression a majority of clinicians opt to keep the patient on continuation treatment for at least 1 year. For two episodes there was less agreement, but for patients with three or more relapses or recurrences most clinicians recommended long-term treatment (Alexopoulos et al, 2001). As with other age groups, it is important to maintain the patient on the same medication regimen that led to remission.

Surprisingly, one would be hard pressed to find any evidence to show that the new range of antidepressants developed over the past 20 years has led to an improved prognosis for late-life depression. However, there is more optimism with respect to prevention. Once a patient has recovered, there is good evidence that ongoing treatment with a tricyclic (Old Age Depression Interest Group, 1993), the SSRI citalopram (Klysner et al, 2002) or a combination of medication with a psychological treatment (Reynolds et al, 1999b) are effective. In a recent trial, sertraline at conventional therapeutic dosage was not effective in preventing relapse in older community-dwelling patients over a period of 2 years (Wilson et al, 2003). Therefore, it cannot be assumed that all antidepressants are equally effective in prophylaxis.

\section{References}

Alexopoulos, G. S., Katz, I. R., Reynolds, C. F., et al (2001) Pharmacotherapy of Depressive Disorders in Older Patients. A Postgraduate Medicine Special Report. White Plains, NY: Expert Knowledge Systems.

Anderson, I. M. (2003) Drug treatment of depression: reflections on the evidence. Advances in Psychiatric Treatment, 9, 11-20.

Anderson, I. M., Nutt, D. J. \& Deakin, J. F. W. (2000) Evidence-based guidelines for treating depressive disorders with antidepressants: a revision of the British Association for Psychopharmacology guidelines. Journal of Psychopharmacology, 14, 3020.

Arean, P. A., Perri, M. G., Nezu, A. M., et al (1993) Comparative effectiveness of social problem-solving therapy and reminiscence therapy as treatment for depression in older adults. Journal of Consulting and Clinical Psychology, 61, 1003-1010.

Ariyo, A. A., Haan, M., Tangen, C. M., et al (2000) Depressive symptoms and risks of coronary heart disease and mortality in elderly Americans. Cardiovascular Health Study Collaborative Research Group. Circulation, 102, 1773-1779.

Baldwin, R. C. (2000) The prognosis of depression. Current Opinions in Psychiatry, 13, 81-85.

Baldwin, R. C. \& O'Brien, J. (2002) Vascular basis of lateonset depressive disorder. British Journal of Psychiatry, 180, 157-160.

Baldwin, R. C., Chiu, E., Katona, C., et al (2002) Guidelines on Depression in Older People: Practising the Evidence. London: Martin Dunitz.

Beekman, A. T., Copeland, J. R. \& Prince, M. J. (1999) Review of community prevalence of depression in later life. British Journal of Psychiatry, 174, 307-311.

Benbow, S., Egan, D., Marriott, A., et al (1990) Using the family life cycle with later life families. Journal of Family Therapy, 12, 321-340.

Carson, A. J., MacHale, S., Allen, K., et al (2000) Depression after stroke and lesion location: a systematic review. Lancet, 356, 122-126.

Cole, M. G. \& Bellavance, F. (1997a) The prognosis of depression in old age. American Journal of Geriatric Psychiatry, 5, 4-14.

Cole, M. G. \& Bellavance, F. (1997b) Depression in elderly medical inpatients: a meta-analysis of outcome. Canadian Medical Association Journal, 157, 1055-1060.

Cuijpers, P. \& Smit, F. (2002) Excess mortality in depression: a meta-analysis of community studies. Journal of Affective Disorders, 72, 227-236.

Garner, J. (2002) Psychodynamic work and older adults. Advances in Psychiatric Treatment, 8, 128-135.

Gill, D. \& Hatcher, S. (1999) Antidepressant drugs in depressed patients who also have a physical illness. Cochrane Library, issue 3. Oxford: Update Software.

Katona, C. (1996) Managing depression in older people. Advances in Psychiatric Treatment, 2, 178-185. 
Katona, C. \& Livingston, G. (2002) How well do antidepressants work in older people? A systematic review of Number Needed to Treat. Journal of Affective Disorders, 69, 47-52.

Kirby, D. \& Ames, D. (2001) Hyponatraemia and selective re-uptake inhibitors in elderly patients. International Journal of Geriatric Psychiatry, 16, 484-493.

Klysner, R., Bent-Hansen, J., Hansen, H. L., et al (2002) Efficacy of citalopram in the prevention of recurrent depression in elderly patients: placebo-controlled study of maintenance therapy. British Journal of Psychiatry, 181, 29-35.

Koenig, H. G., George, L. K., Peterson, B. L., et al (1997) Depression in medically ill hospitalized older adults: prevalence, characteristics, and course of symptoms according to six diagnostic symptoms. American Journal of Psychiatry, 154, 1376-1383.

MacHale, S. (2002) Managing depression in physical illness. Advances in Psychiatric Treatment, 8, 297-305.

Manes, F., Jorge, R., Morcuende, M., et al (2001) A controlled study of repetitive transcranial magnetic stimulation as a treatment of depression in the elderly. International Psychogeriatrics, 13, 225-231.

McCusker, J., Cole, M., Keller, E., et al (1998) Effectiveness of treatments of depression in older ambulatory patients. Archives of Internal Medicine, 158, 705-712.

Mossey, J. M., Knott, K. A., Higgins, M., et al (1996) Effectiveness of a psychosocial intervention, interpersonal counseling, for subdysthymic depression in medically ill elderly. Journal of Gerontology: Medical Sciences, 51A, M172-M178.

Mottram, P. G., Wilson, K. C. M., Ashworth, L., et al (2002) The clinical profile of older patients' response to antidepressants - an open trial of sertraline. International Journal of Geriatric Psychiatry, 17, 574-578.

Mulsant, B. H., Alexopoulos, G. S., Reynolds III, C. F., et al (2001) Pharmacological treatment of depression in older primary care patients: the PROSPECT algorithm International Journal of Geriatric Psychiatry, 16, 585-592.

National Health Service Executive (1996) Psychotherapy Services in England: Review of Strategic Policy. Leeds: NHS Executive.

Old Age Depression Interest Group (1993) How long should the elderly take antidepressants? A double-blind placebocontrolled study of continuation/prophylaxis therapy with dothiepin. British Journal of Psychiatry, 162, 175-182.

Penninx, B. W., Deeg, D. J., van Eijk, J. T., et al (2000) Changes in depression and physical decline in older adults: a longitudinal perspective. Journal of Affective Disorders, 61, 1-12.

Pinquart, M. \& Sorensen, S. (2001) How effective are psychotherapeutic and other psychosocial interventions with older adults? A meta-analysis. Journal of Mental Health and Aging, 7, 207-243.

Poirier, M. F. \& Boyer, P. (1999) Venlafaxine and paroxetine in treatment-resistant depression. Double-blind, randomised comparison. British Journal of Psychiatry, 175, 12-16.

Reynolds III, C. F., Miller, M. D., Pasternak, R. E., et al (1999a) Treatment of bereavement-related major depressive episodes in later life: a controlled study of acute and continuation treatment with nortriptyline and interpersonal psychotherapy. American Journal of Psychiatry, 156, 202208.

Reynolds III, C. F., Frank, E., Perel, J. M., et al (1999b) Nortriptyline and interpersonal psychotherapy as maintenance therapies for recurrent major depression: a randomized controlled trial in patients older than 59 years. $J A M A, \mathbf{2 8 1}, 39-45$.

Rinaldi, P., Mecocci, P., Benedetti, C., et al (2003) Validation of the five-item Geriatric Depression Scale in elderly subjects in three different settings. Journal of the American Geriatric Society, 51, 694-698.

Rost, K., Nutting, P., Smith, J. L., et al (2002) Managing depression as a chronic disease: a randomised trial of ongoing treatment in primary care. BMJ, 325, 934-937.

Schimmel-Spreeuw, A., Linssen, A. C. G. \& Heeren, T. J. (2000) Coping with depression and anxiety: preliminary results of a standardized course for elderly depressed women. International Psychogeriatrics, 12, 77-86.
Simpson, S., Baldwin, R. C., Jackson, A., et al (1998) Is subcortical disease associated with a poor response to antidepressant? Neurological, neuropsychological and neuroradiological findings in late life depression. Psychological Medicine, 28, 1015-1026.

Tew, J. D., Mulsant, B. H., Haskett, R. F., et al (1999) Acute efficacy of ECT in the treatment of major depression in the Old-Old. American Journal of Psychiatry, 156, 1865-1870.

Thompson, L. W., Coon, D. W., Gallagher-Thompson, D., et al (2001) Comparison of desipramine and cognitive/ behavioral therapy in the treatment of elderly outpatients with mild-to-moderate depression. American Journal of Geriatric Psychiatry, 9, 225-240.

Tuma, T. A. (2000) Outcome of hospital-treated depression at 4.5 years. An elderly and a younger adult cohort compared. British Journal of Psychiatry, 176, 224-228.

Unützer, J., Katon, W., Callahan, C., et al (2002) Collaborative care management of late-life depression in the primary care setting. IAMA, 288, 2836-2845.

van Walraven, C., Mamdani, M. \& Wells, P. (2001) Inhibition of serotonin reuptake by antidepressants and upper gastrointestinal bleeding in elderly patients: retrospective cohort study. BMJ, 323, 655-658.

Williams, J. W., Barrett, J., Oxman, T., et al (2000) Treatment of dysthymia and minor depression in primary care: a randomised controlled trial in older adults. JAMA, 284, 1519-1526.

Wilson, K., Mottram, P., Sivanranthan, A., et al (2001) Antidepressant versus placebo for depressed elderly. Cochrane Library, issue 4. Oxford: Update Software.

Wilson, K. C. M., Mottram, P. G., Ashworth, L., et al (2003) Older community residents with depression: long-term treatment with sertraline. Randomised, double-blind, placebo-controlled study. British Journal of Psychiatry, 182, 492-497.

Yesavage, J. A., Brink, T. L., Rose, T. L., et al (1983) Development and validation of a geriatric depression screening scale: preliminary report. Journal of Psychiatric Research, 17, 37-49.

Yohannes, A. M., Roomi, J., Baldwin, R. C., et al (1998) Depression in elderly outpatients with disabling chronic obstructive pulmonary disease. Age and Ageing, 27, 155160.

\section{Multiple choice questions}

1 Depressive disorder in older people:

a is prevalent in 1 in 100

b strongly predicts later dementia

c affects almost half of patients with Parkinson's disease

$\mathrm{d}$ is more common in vascular than in Alzheimer's dementia

e often presents with hypochondriasis.

2 Management principles include:

a symptom improvement as the main goal

b dietary advice

c assessment of self-neglect

d a serum ferritin level

e detailed neuropsychological examination.

3 Treatment:

a always starts with an SSRI

b should be changed if there has been a $25-50 \%$ response at 4 weeks

c with cognitive-behavioural therapy is an effective alternative to antidepressants for moderate depression

$\mathrm{d}$ of mild cases with a combination of medication and a psychological intervention is more effective than either alone

e with ECT is hardly ever effective in the 'old-old'. 
4 Prevention:

a of relapse requires treatment for about 6 months at most

b of recurrence has been demonstrated only for tricyclic antidepressants

c of recurrence after a third episode is an indication for long-term antidepressant treatment

$\mathrm{d}$ of recurrence in moderate-to-severe depression is best achieved by combining an antidepressant with interpersonal therapy

e of depression should include attending to handicap.

\section{MCQ answers}

$\begin{array}{lllllll}1 & & 2 & & 3 & & 4 \\ \text { a F } & \text { a F } & \text { a F } & \text { a F } \\ \text { b F } & \text { b T } & \text { b F } & \text { b F } \\ \text { c T } & \text { c T } & \text { c T } & \text { c T } \\ \text { d T } & \text { d F } & \text { d F } & \text { d T } \\ \text { e T } & \text { e F } & \text { e F } & \text { e T }\end{array}$

\section{Young People and Substance Misuse}

\section{Edited by llana Crome, Hamid Ghodse, Eilish Gilvarry and Paul McArdle}

Substance misuse is one of the most common and serious yet preventable risks to a young person's health and development. This book provides an overview of the consequences of substance misuse, the interventions and services available, and, most importantly, the way forward for improving treatment and services. Young People and Substance Misuse brings international expertise together with a UK health care perspective. It will give the reader an in-depth understanding of the issues as well as suggesting practical solutions to a problem that affects so many aspects of the well-being of teenagers.

This is a book for all those who need to know more about the options for prevention and treatment - teachers, carers, parents, researchers and policy-makers as well as those working in the criminal justice system, social services and mental health care.

The book includes expert analysis of:

- Prevalence and routes to drug use

- Misuse of illicit drugs, alcohol and tobacco

- Parental, family and social influences

- Treatment and prevention strategies.

February 2004, Paperback, 240pp, ISBN 190467101 2, Price $£ 15.00$

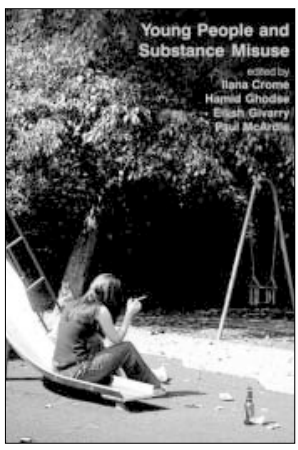

